\title{
Parting ways with routine postoperative chest radiographs: Are we ready to cut the cord?
}

\author{
Nicholas D. Andersen, MD
}

See related article on pages $225-9$.

Should a chest radiograph (CXR) be performed on every patient after heart surgery? The question of whether to perform CXRs routinely or selectively in various intensive care unit patient populations, including postoperative cardiac surgical patients, has been scrutinized for more than 2 decades. ${ }^{1,2}$ In a simple yet elegant study in the current issue of the Journal, Tolsma and colleagues ${ }^{3}$ shed additional light on this question by prospectively testing a selective protocol for postoperative CXR use during the first 24 hours after cardiac surgery with sternotomy at a single center in The Netherlands. Indications for selective (on-demand) CXRs were carefully defined in the article and included difficult central venous access, intra-aortic balloon pump placement, and hemodynamic, ventilatory, or physical examination abnormalities. Tolsma and colleagues ${ }^{3}$ then verified the sensitivity of their selection protocol by performing CXRs on all remaining patients the next morning to screen for any missed findings among the patients who had failed to meet criteria for an ondemand CXR. According to their selection protocol, only 301 of 1102 patients $(27 \%)$ met indications for an ondemand CXR after surgery; of these, 12 patients (4\%) required intervention. Among the remaining 801 patients who did not receive an on-demand CXR on the day of surgery, screening CXRs the next morning identified only 5 patients $(0.6 \%)$ who had missed findings requiring intervention (4 chest tubes for pneumothorax or effusion and 1 transthoracic echocardiogram for widened mediastinum), and none of these patients were harmed as a result of the delay in diagnosis. Thus the selective CXR protocol achieved a $73 \%$ reduction in immediate postoperative CXR use, leading to reduced radiation exposure to patients, a predicted cost saving of $\$ 40,000 / y e a r$, and no apparent increase in adverse events.

From the Division of Cardiovascular and Thoracic Surgery, Department of Surgery, Duke University Medical Center, Durham, NC.

Disclosures: Author has nothing to disclose with regard to commercial support.

Received for publication April 26, 2015; accepted for publication April 28, 2015.

Address for reprints: Nicholas D. Andersen, MD, Division of Cardiovascular and

Thoracic Surgery, Duke University Medical Center, Box 3443, Durham, NC

27710 (E-mail: Nicholas.andersen@duke.edu).

J Thorac Cardiovasc Surg 2015;150:230-1

$0022-5223 / \$ 36.00$

Copyright (c) 2015 by The American Association for Thoracic Surgery

http://dx.doi.org/10.1016/j.jtcvs.2015.04.040
The rationale for compulsory chest imaging after cardiac surgery is to detect and address potentially harmful problems before any clinical manifestations. Similar to other screening tools, for routine CXRs to have

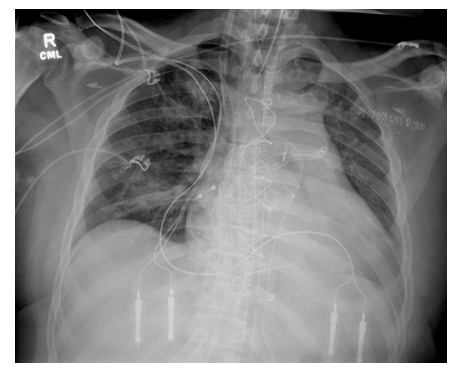
value they must pose minimal risk to the patient, identify important pathology before the onset of signs or symptoms, allow for improved outcomes through early intervention, minimize overtreatment of false-positive findings, and be cost-effective. By most accounts, CXRs fulfill these criteria because they are safe and inexpensive, and the benefits of identifying major problems before acute decompensation (tamponade, tension pneumothorax, etc) are potentially large. As a result, most cardiac surgical centers are willing to accept the costs and small risks of performing routine CXRs on all postoperative cardiac surgical patients. The study by Tolsma and colleagues, however, effectively challenges this practice and suggests that important life-threatening problems are not missed when routine CXRs are abolished and replaced with ondemand CXRs obtained only for defined clinical indications. The goals of minimizing patient exposure to unnecessary imaging and laboratory tests ${ }^{4}$ and of reducing the costs of surgical intervention are clearly laudable, and Tolsma and colleagues ${ }^{3}$ are to be congratulated for their effort.

Despite these convincing findings, certain study limitations may render some centers reluctant to abandon routine postoperative CXRs without further evidence of safety and cost-effectiveness. First, the study by Tolsma and colleagues ${ }^{3}$ does not contain a control group or report objective clinical end points. As a result, although the number of clinically significant missed findings as a consequence of the selective CXR protocol was low, it is unclear whether there was any survival or outcomes benefit or hazard to this protocol relative to patients subjected to a routine CXR protocol. Second, although Tolsma and colleagues ${ }^{3}$ demonstrated in an 1100-patient single-center series that the selective CXR protocol was safe, extrapolating their experience to the general population without a greater sample size may be premature. At present, Tolsma and colleagues ${ }^{3}$ have shown only that the major error rate of their selective CXR protocol is less than 1 in 1100, but how many serious adverse events might be detected if the sample size were to be increased 
to 10,000 , to 20,000 or to the more than 200,000 patients undergoing cardiac surgery yearly in the United States? Even if a serious adverse event occurred only once per 10,000 patients as a result of the selective CXR protocol, the modest cost saving of $\$ 40,000 /$ year per center (or $\$ 36 /$ patient) could easily be erased by a single major patient complication resulting in reoperation, organ failure, or prolonged hospitalization. In the era of fervent attention to quality, safety, and process improvements; public reporting; never events; and the national pursuit of $1 \%$ coronary artery bypass grafting mortality, ${ }^{5}$ many centers may understandably remain hesitant to part ways with the time-honored practice of routine postoperative CXR until proved safe in a larger, multiinstitutional population of cardiac surgical patients.

\section{References}

1. Ganapathy A, Adhikari NK, Spiegelman J, Scales DC. Routine chest x-rays in intensive care units: a systematic review and meta-analysis. Crit Care. 2012;16:R68.

2. Hornick PI, Harris P, Cousins C, Taylor KM, Keogh BE. Assessment of the value of the immediate postoperative chest radiograph after cardiac operation. Ann Thorac Surg. 1995;59:1150-3; discussion 1153-4.

3. Tolsma M, Rijpstra TA, Rosseel PM, Scohy TV, Bentala M, Mulder PG, et al Defining indications for selective chest radiography in the first 24 hours after cardiac surgery. J Thorac Cardiovasc Surg. 2015;150:225-9.

4. Koch CG, Reineks EZ, Tang AS, Hixson ED, Phillips S, Sabik JF III, et al. Contemporary bloodletting in cardiac surgical care. Ann Thorac Surg. 2015;99:779-84.

5. Mack MJ. If this were my last speech, what would I say? Ann Thorac Surg. 2012; 94:1044-52. 